\title{
Analysis of circulating microRNAs in patients with repaired Tetralogy of Fallot with and without heart failure
}

\author{
Masood Abu-Halima ${ }^{1,4^{*}}$, Eckart Meese ${ }^{1}$, Andreas Keller ${ }^{2}$, Hashim Abdul-Khaliq $^{3}$ and Tanja Rädle-Hurst ${ }^{3}$
}

\begin{abstract}
Background: MicroRNAs (miRNAs) are a class of regulatory RNAs that regulate gene expression post-transcriptionally. Little, however, is known on the expression profile of circulating miRNAs in Tetralogy of Fallot (TOF) patients late after surgical repair. In this study, we aimed to identify the specific patterns of circulating miRNAs in blood of patients with repaired, non-syndromic TOF and to assess whether these specific miRNAs may be useful to differentiate patients with and without heart failure.

Methods: SurePrint ${ }^{\mathrm{TM}} 8 \times 60 \mathrm{~K}$ Human v16 miRNA arrays were used to determine miRNA expression profiles in 15 healthy controls and 37 patients after TOF repair of whom 3 had symptomatic right heart failure. The expression levels of selected miRNAs have been validated by quantitative reverse transcription polymerase chain reaction (RT-qPCR). Enrichment analyses of altered miRNA expression were predicted using bioinformatic tools.

Results: Compared with healthy controls, a total of 49,58 and 77 miRNAs were found to be significantly altered in TOF patients (TOF-all), TOF patients with (TOF-HF) and without symptomatic right heart failure (TOF-noHF) (>2.0-fold change, adjusted $P<0.05)$, respectively. Three miRNAs namely miR-181d-5p, miR-206 and miR-625-5p were validated by RT-qPCR in all TOF groups. The area under the receiver operating characteristic curve (AUC) for miR-181d-5p, miR-206 and miR-625-5p were 0.987, 0.993 and 0.769 in TOF-all and 0.990, 0.994 and 0.749 in TOF-noHF, respectively. Moreover, expression levels of miR-625-5p, miR-1233-3p and miR-421 were lower in TOF-HF compared to TOF-noHF $(P=0.012)$.

Conclusions: Altered expression levels of circulating miRNAs were found in TOF patients late after surgical repair and are different to those seen in the right ventricular myocardium of infants with TOF. Expression levels of miR-421, miR1233-3p and miR-625-5p are lower in TOF patients with symptomatic right heart failure and thus may indicate disease progression in these patients.
\end{abstract}

Keywords: MicroRNA, Heart failure, Congenital heart defects, Tetralogy of Fallot

\section{Background}

Tetralogy of Fallot (TOF) is the most frequent cyanotic congenital heart defect with a prevalence of $5-7 \%$ per 10,000 live births [1]. It is characterized by varying degrees of right ventricular outflow tract (RVOT) obstruction and a large ventricular septal defect requiring surgical repair within the first years of life. After

\footnotetext{
*Correspondence: masood@daad-alumni.de

${ }^{4}$ Department of Human Genetics, Saarland University Medical Center,

Kirrberger Straße 100, 66421 Homburg/Saar, Germany

Full list of author information is available at the end of the article
}

surgical repair, residual lesions such as restenosis of the RVOT or pulmonary regurgitation as well as right or left ventricular dysfunction may occur during long-term follow-up [2-6]. MicroRNAs (miRNAs) are a class of noncoding RNAs of approximately 22 nucleotides in length that regulate gene expression post-transcriptionally via sequence-specific interaction with the $3^{\prime}$ UTR of target mRNAs, resulting in inhibition of translation and/or mRNA degradation [7]. Studies have shown that dysfunctions of miRNAs are associated with congenital heart disease (CHD) [8], including TOF [9-12]. These studies 
have highlighted the alteration of miRNA profile in the myocardial tissue. Recently, alterations in miRNA expression levels in ROVT myocardial tissue of infants with TOF have been identified [9, 10]. Specifically, O'Brien et al. [10] identified 61 miRNAs with significantly altered expression levels and Bittel et al. [9] found that miR-421 exhibited the most significant expressed miRNA in the RVOT myocardial tissue of infants with TOF. Another study by Liang et al. [13] explored the role of miRNA in TOF patients and identified 75 miRNAs with altered expression levels. Among the altered miRNAs, miR940 was the most down-regulated one [13]. Apart from their importance in myocardial tissue, miRNAs have also been detected in blood of patients with various cardiovascular diseases, offering the possibility to use them as potential biomarkers [14-18]. In adult patients with dyspnea, circulating miR-423-5p has been identified as a potential biomarker that can distinguish patients with left-sided heart failure from those with other causes of dyspnea [19]. However, this miRNA failed as a biomarker in patients after atrial switch operation for transposition of the great arteries [20]. In addition, Lai et al. identified 23 miRNAs in patients late after atrial switch operation for complete transposition of the great arteries (TGA), of which miR-18a and miR-486-5p were negatively related to the systolic function of the systemic right ventricle [16]. In TOF patients, however, investigations of circulating miRNAs are still lacking. With this study, we aim to identify the specific patterns of circulating miRNAs in blood of patients with repaired, non-syndromic TOF and to assess whether these specific miRNAs may be useful to differentiate patients with and without heart failure.

\section{Methods}

\section{Patients and blood collection}

A total of 37 consecutive patients late after surgical repair of non-syndromic TOF visiting our outpatient clinic specialized in the treatment of adults with congenital heart disease were enrolled in the study and compared to 15 age-matched healthy controls. In all healthy controls, a physical examination including measurement of blood pressure and transcutaneous oxygen saturation at rest as well as two-dimensional echocardiography were performed to verify the absence of any heart abnormality. Mean age of TOF patients and controls was $30.2 \pm 10.8$ years. In all TOF patients, two- and threedimensional echocardiography was performed using a Vivid $^{\mathrm{TM}}$ E9 Ultrasound System (GE Healthcare, Horten, Norway) to assess right and left ventricular dimensions as well as systolic biventricular function. In all controls and patients, venous blood from the cubital vein was drawn and $2.5 \mathrm{ml}$ collected into PAXgene ${ }^{\mathrm{TM}}$ blood tubes (Becton-Dickinson, Heidelberg, Germany) shortly after echocardiographic evaluation. In the patient group, venous blood samples were drawn to determine the routine laboratory parameters as well as the concentrations of NT-proBNP and high sensitive troponin T (hsTNT) that were measured using electrochemiluminescence sandwich immunoassays namely Cobas ${ }^{\circledR}$ proBNP II and Elecsys ${ }^{\circledR}$ Troponin T high sensitive by Roche Diagnostics, Basel, Switzerland. All PAXgene blood tubes were stored at room temperature for $24 \mathrm{~h}$ to ensure complete lysis of the blood cells, then stored at $-20^{\circ} \mathrm{C}$ for several days and finally transferred to $-80^{\circ} \mathrm{C}$ for long-term storage until RNA isolation. Subjects were recruited and evaluated in accordance with relevant guidelines and regulations following the approval of the local ethics committee (Ethical vote No. 73/09). All participants or their legal guardians gave written informed consent before enrolment.

\section{RNA isolation and quality assessment}

Total RNA including miRNAs from venous blood collected into PAXgene tubes was isolated using PAXgene Blood miRNA Kit on the QIAcube ${ }^{\mathrm{TM}}$ robot (Qiagen, Hilden, Germany) following the manufacturer's recommendations and included DNase I treatment (Qiagen, Hilden, Germany). To confirm the absence of genomic DNA contamination, a conventional PCR with exon spanning primers for GAPDH was performed [21]. The concentration of isolated total RNA, including miRNAs was measured using NanoDrop ND-2000 spectrophotometer (Thermo Fisher Scientific, Massachusetts, USA). RNA purity was estimated by examining the OD 260/280 and the OD 260/230 ratios. The RNA integrity was assessed by Agilent 2100 Bioanalyzer using the RNA Nano 6000 Kit according to the manufacturer's instructions (Agilent Technologies, California, USA).

\section{Analysis of circulating miRNAs by microarray}

MiRNA expression analysis was performed according to the manufacturer's instructions using SurePrint ${ }^{\mathrm{TM}}$ $8 \times 60 \mathrm{~K}$ Human v16 miRNA microarrays (Agilent Technologies, California, USA). These microarrays contain $\sim 40$ replicates for each probe complement to each of the 1205 mature miRNAs of miRBase v16. These probes act in concert to measure the miRNA of interest, and the data are combined later during software analysis. All probes are randomly distributed on the array, and cross hybridization is prevented by the addition of a $\mathrm{G}$ residue and a hairpin at the 50 end of the probe. Microarray hybridizations were done following the manufacturer's recommendations. In brief, a total of 100 ng total RNA from each sample was dephosphorylated by incubation with calf intestinal phosphatase at $37^{\circ} \mathrm{C}$ for $30 \mathrm{~min}$ and denatured with the use of $100 \%$ dimethyl sulfoxide at $100{ }^{\circ} \mathrm{C}$ for $7 \mathrm{~min}$. Samples were labeled with $\mathrm{pCp}-\mathrm{Cy} 3$ 
with the use of T4 ligase at $16^{\circ} \mathrm{C}$ incubation for $2 \mathrm{~h}$. Each labeled RNA sample was hybridized onto an individual sub-array of the $8 \times 60 \mathrm{~K}$ format Agilent miRNA microarray slide, with each array containing probes for 1205 human miRNAs according to miRBase v16. Then the microarrays were loaded and incubated at $55{ }^{\circ} \mathrm{C}$ for $20 \mathrm{~h}$ with rotation. After two washing steps, the arrays were dried and scanned using the Agilent Microarray Scanner at 3 microns in double path mode. Data was acquired using Agilent AGW Feature Extraction software version 10.10.11 (Agilent Technologies, California, USA).

\section{Analysis of circulating miRNAs by RT-qPCR}

RT-qPCR validation analysis was performed according to the manufacturer's instructions using EPIK $^{\mathrm{TM}}$ miRNA Select Assays (Bioline, London, UK) to validate the results obtained from miRNA microarray initial survey experiments. In brief, 100 ng RNA was converted into cDNA using the EPIK ${ }^{\mathrm{TM}}$ miRNA RT kit (Bioline, London, UK). The cDNA was diluted 1:10 and $4 \mu \mathrm{l}$ of cDNA was mixed with $10 \mu \mathrm{l} 2 \mathrm{X}$ SensiSMART ${ }^{\mathrm{TM}}$ PCR Master Mix, $2 \mu \mathrm{l}$ PCR Primer for 10 miRNAs (miR-181d-5p, miR142-5p, miR-140-3p, miR-1233-3p, miR-183-5p, miR206, miR-339-5p, miR-421 and miR-625-5p) and U6 snRNA as an endogenous control (Bioline, London, UK) in a total volume of $20 \mu$ l. All RT-qPCR experiments were performed using the QIAgility ${ }^{\mathrm{TM}}$ automated PCR setup robot (Qiagen, Hilden, Germany) before performing RTqPCR analysis on a StepOnePlus ${ }^{\mathrm{TM}}$ Real-Time PCR system (Applied Biosystems, Foster City, USA). The melting curve analysis was used to control the specificity of RTqPCR products.

\section{Statistical analysis}

For statistical analysis, the freely available $\mathrm{R}$ statistical environment v.2.14.2 was used to analyze the differences in miRNA expression in patient groups compared to healthy controls. Raw data generated by Agilent Feature Extraction image analysis software was quantile normalized. A significance level of miRNAs was analyzed by applying an unpaired two-tailed t test and area under the receiver operating characteristic curve (AUC) values for each miRNA were computed. The relative quantitative method of $2^{-\Delta \Delta C q}$ was used to measure the dynamic change of specifically selected miRNAs [22]. In detail, first, the threshold cycle $(\mathrm{Ct})$ value for each sample was determined. Next, the Delta $\mathrm{Ct}(\Delta \mathrm{Ct})$ value between the $\mathrm{Ct}$ value of the target miRNA and the $\mathrm{Ct}$ value of the endogenous control was calculated using equation: $\Delta \mathrm{Ct}=\mathrm{Ct}$ (target miRNA) $-\mathrm{Ct}$ (endogenous control). In the next steps, the $\Delta \Delta \mathrm{Ct}$ value and the normalized target expression were calculated: $\Delta \Delta \mathrm{Ct}=\Delta \mathrm{Ct}$ (sample [e.g., TOF sample]) $-\Delta \mathrm{Ct}$ (control [e.g., healthy controls].
Finally, the fold change was calculated using the equation $2^{-\Delta \Delta \mathrm{Ct}}$. The analyses of clinical data and their correlation to miRNA expression levels were performed using the statistical software package SPSS (SPSS version 19; SPSS Inc., Chicago, Illinois). Data are presented as the mean \pm standard deviation or medians (interquartile range) as appropriate. The effect of miRNAs on target genes and networks has been evaluated using miRTargetLink [23].

\section{Results}

\section{Patient characteristics}

Of the 37 patients enrolled in the study 22 were female and 15 were male. Three of them presented with peripheral edema, hepatomegaly and significant weight gain over a short period of time indicating symptomatic right heart failure. The other 34 patients were in a clinically stable condition without any clinical signs of right heart failure. Clinical characteristics of TOF patients with and without symptomatic right heart failure are displayed in Table 1. In TOF patients with symptomatic right heart failure, NYHA class, right and left ventricular volumes were significantly elevated whereas measures of systolic ventricular function such as ejection fraction or velocity time integral above the aortic valve were significantly reduced. Moreover, NT-proBNP levels were also significantly higher in these patients.

\section{Differentially expressed miRNAs}

Using the high-throughput SurePrint G3 Human v16 miRNA microarray platform, we measured the expression of 1205 human mature miRNAs of miRBase v16. Following background correction and quantile normalization, expression levels of circulating miRNAs were screened in a total of 21 TOF patients (TOF-all), of whom 3 had symptomatic right heart failure (TOF-HF, $\mathrm{n}=3$ ) and 18 had no signs of right heart failure (TOF-noHF, $\mathrm{n}=18)$ as well as 15 healthy controls. Next, we performed an unpaired $t$ test to identify those miRNAs that showed a differential expression in TOF-all compared to healthy controls as well as in TOF-noHF and TOF-HF, each also compared to an age-matched healthy control group (Additional file 1: Table S1). We next considered those miRNAs as significantly differentially expressed that showed a fold change of $>2.0$ between considered groups and a $P$ value $<0.05$ in an unpaired $t$ test (Table 1). In total, 49 significantly deregulated miRNAs were identified in TOF-all patients (27 up-regulated and 22 down-regulated) (Table $2 \mathrm{a}, \mathrm{P}<0.05$, FDR adjusted), 58 significantly altered miRNAs in the TOF-noHF subset (33 up-regulated and 25 down-regulated) (Table $2 \mathrm{~b}$, $\mathrm{P}<0.05$, FDR adjusted) and 77 significantly deregulated miRNAs in the TOF-HF group (42 up-regulated and 35 
Table 1 Characteristics of TOF patients with (TOF-HF) and without symptomatic right heart failure (TOF-noHF)

\begin{tabular}{|c|c|c|c|c|c|}
\hline Variables & Controls $(n=15)$ & All patients $(n=37)$ & TOF-noHF $(n=34)$ & TOF-HF $(n=3)$ & $P$ value* \\
\hline Age at follow-up (years) & $30.2 \pm 10.8$ & $30.2 \pm 10.8$ & $29.9 \pm 10.7$ & $33.0 \pm 13.9$ & NS \\
\hline Follow-up time (years) & - & $24.8 \pm 8.9$ & $25.1 \pm 8.1$ & $24.7 \pm 17.7$ & NS \\
\hline Age at primary corrective surgery (years) & - & $4.9 \pm 3.5$ & $4.6 \pm 3.5$ & $8.0 \pm 2.6$ & 0.079 \\
\hline Prior BT shunt (\%) & - & 20/37 (54.1) & 19/34 (55.9) & $1 / 3(33.3)$ & NS \\
\hline $\begin{array}{l}\text { Presence of pulmonary bioprosthetic valve or } \\
\text { homograft (\%) }\end{array}$ & - & $15 / 37(40.5)$ & $14 / 34(41.2)$ & $1 / 3(33.3)$ & NS \\
\hline $\begin{array}{l}\text { Presence of moderate to severe residual pulmo- } \\
\text { nary regurgitation (\%) }\end{array}$ & - & $8 / 37(21.6)$ & $6 / 34(17.6)$ & $2 / 3(66.7)$ & NS \\
\hline $\begin{array}{l}\text { Residual peak systolic pressure gradient across } \\
\text { RVOT }>40 \mathrm{mmHg}(\%)\end{array}$ & - & $11 / 37(29.7)$ & 10/34 (29.4) & $1 / 3(33.3)$ & NS \\
\hline NYHA functional class & $1.1 \pm 0.3$ & $1.3 \pm 0.6$ & $1.1 \pm 0.3$ & $2.7 \pm 0.6$ & $<0.001$ \\
\hline Systolic blood pressure (mmHg) & $119.7 \pm 10.8$ & $122.2 \pm 13.2$ & $122.7 \pm 13.7$ & $115.7 \pm 2.5$ & NS \\
\hline Diastolic blood pressure $(\mathrm{mmHg})$ & $66.7 \pm 6.2$ & $70.4 \pm 8.4$ & $70.0 \pm 8.6$ & $75.0 \pm 3.6$ & NS \\
\hline Transcutaneous oxygen saturation at rest (\%) & $98.1 \pm 1.1$ & $97.7 \pm 1.4$ & $97.7 \pm 1.4$ & $97.3 \pm 1.5$ & NS \\
\hline $\begin{array}{l}\text { Presence of any medication such as } \beta \text {-blocker, } \\
\text { ACE inhibitor/AT1 blocker or diuretics (\%) }\end{array}$ & - & 14/37 (37.8) & $11 / 34(32.2)$ & $3 / 3(100.0)$ & 0.047 \\
\hline Ejection fraction of RV (\%) & $62.9 \pm 3.7$ & $53.5 \pm 8.5$ & $55.5 \pm 5.9$ & $33.3 \pm 2.3$ & 0.005 \\
\hline Enddiastolic volume of RV (ml/m² BSA) & $28.7 \pm 8.5$ & $61.8 \pm 29.2$ & $55.9 \pm 22.3$ & $122.4 \pm 23.9$ & 0.007 \\
\hline Endsystolic volume of RV (ml/m² BSA) & $10.6 \pm 3.35$ & $30.6 \pm 21.1$ & $25.5 \pm 12.2$ & $84.1 \pm 21.4$ & 0.006 \\
\hline Ejection fraction of LV (\%) & $62.6 \pm 3.2$ & $58.4 \pm 9.9$ & $60.6 \pm 6.0$ & $33.1 \pm 11.8$ & 0.005 \\
\hline Enddiastolic volume of $\mathrm{LV}\left(\mathrm{ml} / \mathrm{m}^{2} \mathrm{BSA}\right)$ & $62.5 \pm 13.9$ & $58.5 \pm 17.3$ & $56.0 \pm 14.4$ & $87.0 \pm 25.0$ & 0.014 \\
\hline Endsystolic volume of LV (ml/m² BSA) & $23.5 \pm 6.0$ & $27.2 \pm 13.0$ & $24.5 \pm 8.1$ & $57.9 \pm 20.3$ & 0.005 \\
\hline VTI above aortic valve $(\mathrm{cm})$ & $27.4 \pm 3.5$ & $22.6 \pm 4.6$ & $23.4 \pm 3.8$ & $14.0 \pm 3.6$ & 0.008 \\
\hline Creatinine (mg/dl) & ND & $0.83(0.69-0.90)$ & $0.82(0.69-0.88)$ & $0.92(0.70-1.36)$ & NS \\
\hline Estimated glomerular filtration rate (ml/min) & ND & $106.2 \pm 19.8$ & $107.8 \pm 15.6$ & $89.4 \pm 49.6$ & NS \\
\hline NT-proBNP (pg/ml) & ND & $146.0(74.8-236.2)$ & $139.6(65.6-215.2)$ & $1747.0(1531.0-2939.5)$ & 0.005 \\
\hline High sensitive troponin T (pg/ml) & ND & $4.5(3.0-6.0)$ & $4.0(3.0-6.0)$ & $6.0(6.0-9.5)$ & 0.057 \\
\hline
\end{tabular}

Mean \pm standard deviation or median (interquartile range) are used

TOF Tetralogy of Fallot, BT Blalock-Taussig, RVOT right ventricular outflow tract, NYHA New York Heart Association, RV right ventricle, BSA body surface area, LV left ventricle, VTI velocity time integral, ND not determined, NS not significant

* Patients with compared to those without symptomatic right heart failure (TOF-HF versus TOF-noHF)

down-regulated miRNAs) (Table $2 \mathrm{c}, \mathrm{P}<0.05$ ), each compared to an age-matched healthy controls. Using hierarchic clustering with the euclidian distance measure, we analyzed how the TOF patients and controls were related to each other. For this task, we selected the 50 miRNAs with the highest variance of miRNA levels out of the 1205 miRNAs. Additional file 2: Figure S1 shows the resulting heatmap of the hierarchic clustering. We observed two distinct clusters between TOF-HF and matched controls Additional file 2: Figure S1A. The first cluster contains mostly controls and the second most of the TOF-HF patients. A more detailed distinction between the TOFnoHF and TOF-all subset, each matched to controls based on the clustering dendrogram were, however, not conclusive (Additional file 2: Figure S1B, C).

\section{Validation of candidate miRNAs by RT-qPCR}

Using RT-qPCR, the validation of microarray data was performed to re-examine the expression level of
9 miRNAs, namely miR-181d-5p, miR-142-5p, miR140-3p, miR-1233-3p, miR-183-5p, miR-206, miR339-5p, miR-421 and miR-625-5p by RT-qPCR. These miRNAs were chosen based on their differential expression level in each patient group and matched controls and based on their known associations with cardiovascular diseases [9, 24-34]. In detail, we selected two miRNAs with high fold changes among the up-regulated (miR625-5p and miR-183-5p) and four miRNAs with high fold changes among the down-regulated ones (miR-181d-5p, miR-206, miR-142-5p and miR-339-5p). These miRNAs were shared in the three comparisons. In addition, we selected three miRNAs (miR-1233-3p, miR-140-3p and miR-421) with low or moderate expression levels in the three comparisons and miR-421 that had been identified in TOF [9]. In the first validation, a total of 37 TOF-all patients and 15 healthy controls were included in the analysis. The RT-qPCR showed the same direction of expression changes as the microarray analysis 
Table 2 The greatest fold change in miRNA expression levels in the blood of patient with TOF-all, TOF-noHF, and TOF-HF compared to age matched healthy controls as determined by microarray (unpaired two-tailed $t$ test, $>2.0$-fold difference, and FDR, $P<0.05$ )

\begin{tabular}{|c|c|c|c|c|c|}
\hline miRNA & $P$ value & Corrected $P$ value & Fold change & Regulation & AUC \\
\hline \multicolumn{6}{|c|}{ A) TOF-all patients $(n=21)$ compared to matched healthy controls $(n=15)$} \\
\hline hsa-miR-1231 & 0.000379 & 0.001621 & 6.94 & Up & 0.83 \\
\hline hsa-miR-144* & 0.000248 & 0.001198 & 4.82 & Up & 0.83 \\
\hline hsa-miR-505* & 0.000557 & 0.002201 & 4.61 & Up & 0.83 \\
\hline hsa-miR-625 & 0.007441 & 0.017478 & 3.43 & Up & 0.77 \\
\hline hsa-miR-15b & 0.000412 & 0.001738 & 3.03 & Up & 0.83 \\
\hline hsa-miR-218-1* & 0.001196 & 0.003935 & 2.94 & Up & 0.87 \\
\hline hsa-miR-214 & 0.009782 & 0.021589 & 2.93 & Up & 0.75 \\
\hline hsa-let-7a & 0.000013 & 0.000158 & 2.66 & Up & 0.91 \\
\hline hsa-let-7f & 0.000643 & 0.002493 & 2.46 & Up & 0.84 \\
\hline hsa-miR-20b & 0.000040 & 0.000335 & 2.41 & Up & 0.88 \\
\hline hsa-miR-1274b & 0.000002 & 0.000056 & 2.38 & Up & 0.92 \\
\hline hsa-miR-218-2* & 0.000000 & 0.000016 & 2.31 & Up & 0.95 \\
\hline hsa-let-7g & 0.000008 & 0.000120 & 2.29 & Up & 0.90 \\
\hline hsa-miR-1274a & 0.000138 & 0.000775 & 2.27 & Up & 0.86 \\
\hline hsa-let-7d & 0.000012 & 0.000151 & 2.26 & Up & 0.92 \\
\hline hsa-miR-1233 & 0.000100 & 0.000613 & 2.23 & Up & 0.83 \\
\hline hsa-miR-183 & 0.000221 & 0.001107 & 2.21 & Up & 0.89 \\
\hline hsa-miR-20a & 0.000099 & 0.000609 & 2.21 & Up & 0.86 \\
\hline hsa-miR-1288 & 0.000019 & 0.000195 & 2.20 & Up & 0.89 \\
\hline hsa-miR-138-1* & 0.000006 & 0.000106 & 2.19 & Up & 0.90 \\
\hline hsa-miR-4286 & 0.003120 & 0.008467 & 2.18 & Up & 0.75 \\
\hline hsa-miR-3198 & 0.000335 & 0.001482 & 2.12 & Up & 0.83 \\
\hline hsa-miR-1181 & 0.000180 & 0.000939 & 2.09 & Up & 0.85 \\
\hline hsa-miR-34c-3p & 0.000070 & 0.000488 & 2.07 & Up & 0.83 \\
\hline hsa-miR-3690 & 0.000000 & 0.000010 & 2.05 & Up & 0.99 \\
\hline hsa-miR-3125 & 0.000161 & 0.000862 & 2.04 & Up & 0.83 \\
\hline hsa-let-7b & 0.011673 & 0.025119 & 2.00 & Up & 0.73 \\
\hline hsa-miR-181d & 0.000014 & 0.000164 & 5.52 & Down & 0.05 \\
\hline hsa-miR-3653 & 0.002113 & 0.006271 & 4.28 & Down & 0.21 \\
\hline hsa-miR-206 & 0.000000 & 0.000018 & 2.82 & Down & 0.07 \\
\hline hsa-miR-3201 & 0.006986 & 0.016505 & 2.69 & Down & 0.16 \\
\hline hsa-miR-142-5p & 0.000475 & 0.001951 & 2.60 & Down & 0.19 \\
\hline hsa-miR-181b & 0.000777 & 0.002851 & 2.56 & Down & 0.17 \\
\hline hsa-miR-194 & 0.000000 & 0.000016 & 2.49 & Down & 0.06 \\
\hline hsa-miR-339-5p & 0.000000 & 0.000016 & 2.43 & Down & 0.03 \\
\hline hsa-miR-26a & 0.002104 & 0.006259 & 2.36 & Down & 0.20 \\
\hline hsa-miR-1268 & 0.011120 & 0.024188 & 2.33 & Down & 0.22 \\
\hline hsa-miR-595 & 0.000358 & 0.001548 & 2.31 & Down & 0.17 \\
\hline hsa-miR-151-3p & 0.000000 & 0.000016 & 2.20 & Down & 0.04 \\
\hline hsa-miR-4271 & 0.000012 & 0.000155 & 2.19 & Down & 0.10 \\
\hline hsa-miR-1307 & 0.002101 & 0.006259 & 2.16 & Down & 0.25 \\
\hline hsa-miR-222 & 0.000258 & 0.001225 & 2.16 & Down & 0.14 \\
\hline hsa-miR-30c & 0.000058 & 0.000432 & 2.15 & Down & 0.13 \\
\hline hsa-miR-620 & 0.000001 & 0.000034 & 2.11 & Down & 0.07 \\
\hline hsa-miR-623 & 0.000312 & 0.001418 & 2.09 & Down & 0.12 \\
\hline hsa-miR-30b & 0.000336 & 0.001482 & 2.04 & Down & 0.15 \\
\hline hsa-miR-3689b* & 0.000000 & 0.000020 & 2.04 & Down & 0.03 \\
\hline
\end{tabular}


Table 2 continued

\begin{tabular}{|c|c|c|c|c|c|}
\hline miRNA & $P$ value & Corrected $P$ value & Fold change & Regulation & AUC \\
\hline hsa-miR-186 & 0.009757 & 0.021573 & 2.03 & Down & 0.29 \\
\hline hsa-miR-191 & 0.000000 & 0.000021 & 2.00 & Down & 0.06 \\
\hline \multicolumn{6}{|c|}{ B) TOF-noHF patients ( $n=18$ ) compared to matched healthy controls $(n=15)$} \\
\hline hsa-miR-1231 & 0.000171 & 0.000930 & 8.72 & Up & 0.86 \\
\hline hsa-miR-144* & 0.000156 & 0.000861 & 7.39 & Up & 0.85 \\
\hline hsa-miR-505* & 0.000551 & 0.002170 & 4.54 & Up & 0.83 \\
\hline hsa-miR-214 & 0.009722 & 0.021817 & 3.33 & Up & 0.76 \\
\hline hsa-miR-625 & 0.014818 & 0.031162 & 3.23 & Up & 0.76 \\
\hline hsa-miR-218-1* & 0.000048 & 0.000374 & 3.18 & Up & 0.95 \\
\hline hsa-miR-15b & 0.000582 & 0.002270 & 3.00 & Up & 0.82 \\
\hline hsa-miR-1233 & 0.000043 & 0.000353 & 2.91 & Up & 0.86 \\
\hline hsa-let-7a & 0.000011 & 0.000160 & 2.66 & Up & 0.92 \\
\hline hsa-miR-34c-3p & 0.000112 & 0.000674 & 2.61 & Up & 0.85 \\
\hline hsa-let-7f & 0.000702 & 0.002621 & 2.58 & Up & 0.84 \\
\hline hsa-miR-218-2* & 0.000000 & 0.000019 & 2.58 & Up & 0.96 \\
\hline hsa-miR-138-1* & 0.000011 & 0.000160 & 2.48 & Up & 0.90 \\
\hline hsa-miR-20b & 0.000262 & 0.001258 & 2.48 & Up & 0.86 \\
\hline hsa-miR-20a & 0.000391 & 0.001640 & 2.48 & Up & 0.84 \\
\hline hsa-miR-1181 & 0.000394 & 0.001643 & 2.34 & Up & 0.85 \\
\hline hsa-miR-4286 & 0.001466 & 0.004576 & 2.32 & Up & 0.78 \\
\hline hsa-miR-1274b & 0.000003 & 0.000076 & 2.31 & Up & 0.91 \\
\hline hsa-let-7d & 0.000019 & 0.000225 & 2.24 & Up & 0.91 \\
\hline hsa-miR-3690 & 0.000000 & 0.000019 & 2.23 & Up & 0.99 \\
\hline hsa-miR-1292 & 0.001251 & 0.004106 & 2.21 & Up & 0.75 \\
\hline hsa-miR-4258 & 0.000014 & 0.000189 & 2.20 & Up & 0.91 \\
\hline hsa-miR-1274a & 0.000381 & 0.001627 & 2.18 & Up & 0.85 \\
\hline hsa-miR-1288 & 0.000089 & 0.000575 & 2.16 & Up & 0.87 \\
\hline hsa-miR-183 & 0.000371 & 0.001597 & 2.11 & Up & 0.87 \\
\hline hsa-miR-4319 & 0.000039 & 0.000333 & 2.10 & Up & 0.86 \\
\hline hsa-let-7g & 0.000031 & 0.000290 & 2.08 & Up & 0.90 \\
\hline hsa-miR-124* & 0.000023 & 0.000235 & 2.06 & Up & 0.94 \\
\hline hsa-miR-887 & 0.000004 & 0.000079 & 2.04 & Up & 0.91 \\
\hline hsa-miR-921 & 0.000003 & 0.000067 & 2.02 & Up & 0.90 \\
\hline hsa-miR-3907 & 0.000430 & 0.001761 & 2.02 & Up & 0.84 \\
\hline hsa-miR-196b* & 0.000000 & 0.000019 & 2.01 & Up & 0.96 \\
\hline hsa-let-7b & 0.006964 & 0.016423 & 2.01 & Up & 0.76 \\
\hline hsa-miR-181d & 0.000015 & 0.000204 & 5.34 & Down & 0.06 \\
\hline hsa-miR-3653 & 0.005185 & 0.012829 & 3.99 & Down & 0.23 \\
\hline hsa-miR-206 & 0.000001 & 0.000035 & 2.88 & Down & 0.07 \\
\hline hsa-miR-194 & 0.000000 & 0.000019 & 2.70 & Down & 0.06 \\
\hline hsa-miR-3201 & 0.003859 & 0.010042 & 2.67 & Down & 0.14 \\
\hline hsa-miR-181b & 0.000658 & 0.002515 & 2.62 & Down & 0.17 \\
\hline hsa-miR-339-5p & 0.000000 & 0.000019 & 2.51 & Down & 0.03 \\
\hline hsa-miR-26a & 0.001287 & 0.004168 & 2.38 & Down & 0.19 \\
\hline hsa-miR-595 & 0.000403 & 0.001676 & 2.36 & Down & 0.17 \\
\hline hsa-miR-151-3p & 0.000000 & 0.000019 & 2.28 & Down & 0.04 \\
\hline hsa-miR-1307 & 0.001320 & 0.004219 & 2.24 & Down & 0.23 \\
\hline hsa-miR-3689b* & 0.000003 & 0.000069 & 2.15 & Down & 0.03 \\
\hline hsa-miR-191 & 0.000001 & 0.000025 & 2.15 & Down & 0.06 \\
\hline
\end{tabular}


Table 2 continued

\begin{tabular}{|c|c|c|c|c|c|}
\hline miRNA & $P$ value & Corrected $P$ value & Fold change & Regulation & AUC \\
\hline hsa-miR-142-5p & 0.001336 & 0.004247 & 2.14 & Down & 0.21 \\
\hline hsa-miR-222 & 0.000341 & 0.001515 & 2.12 & Down & 0.15 \\
\hline hsa-miR-620 & 0.000001 & 0.000035 & 2.11 & Down & 0.06 \\
\hline hsa-miR-3129 & 0.000021 & 0.000234 & 2.08 & Down & 0.09 \\
\hline hsa-miR-186 & 0.025390 & 0.049030 & 2.08 & Down & 0.33 \\
\hline hsa-miR-30c & 0.000279 & 0.001306 & 2.07 & Down & 0.16 \\
\hline hsa-miR-4271 & 0.000009 & 0.000143 & 2.06 & Down & 0.10 \\
\hline hsa-miR-140-3p & 0.000004 & 0.000079 & 2.06 & Down & 0.05 \\
\hline hsa-miR-190b & 0.000024 & 0.000241 & 2.05 & Down & 0.08 \\
\hline hsa-miR-623 & 0.000389 & 0.001640 & 2.04 & Down & 0.13 \\
\hline hsa-miR-1262 & 0.000000 & 0.000020 & 2.03 & Down & 0.04 \\
\hline hsa-miR-30d & 0.000018 & 0.000218 & 2.01 & Down & 0.10 \\
\hline \multicolumn{6}{|c|}{ C) TOF-HF patients $(n=3)$ compared to matched healthy controls $(n=3)$} \\
\hline hsa-miR-625 & 0.000868 & 0.040212 & 11.77 & Up & \\
\hline hsa-miR-183* & 0.041803 & 0.150816 & 6.16 & Up & \\
\hline hsa-miR-183 & 0.016584 & 0.093166 & 6.08 & Up & \\
\hline hsa-miR-505* & 0.020963 & 0.105695 & 5.66 & Up & \\
\hline hsa-miR-214 & 0.000609 & 0.032735 & 4.60 & Up & \\
\hline hsa-miR-30e* & 0.015156 & 0.089838 & 4.59 & Up & \\
\hline hsa-let-7d & 0.003245 & 0.062067 & 4.44 & Up & \\
\hline hsa-miR-340* & 0.045108 & 0.154000 & 3.94 & Up & \\
\hline hsa-miR-28-5p & 0.009640 & 0.081007 & 3.81 & Up & \\
\hline hsa-miR-15b & 0.000038 & 0.015689 & 3.81 & Up & \\
\hline hsa-miR-151-5p & 0.000976 & 0.040600 & 3.77 & Up & \\
\hline hsa-let-7a & 0.043951 & 0.153067 & 3.51 & Up & \\
\hline hsa-miR-1271 & 0.001328 & 0.044227 & 3.34 & Up & \\
\hline hsa-miR-192 & 0.001655 & 0.044227 & 3.30 & Up & \\
\hline hsa-miR-20b & 0.011702 & 0.083679 & 3.03 & Up & \\
\hline hsa-miR-23b & 0.001013 & 0.040600 & 3.02 & Up & \\
\hline hsa-miR-148b & 0.014520 & 0.089728 & 2.95 & Up & \\
\hline hsa-miR-339-3p & 0.006304 & 0.071105 & 2.89 & Up & \\
\hline hsa-let-7g & 0.016852 & 0.093166 & 2.85 & Up & \\
\hline hsa-miR-660 & 0.010161 & 0.081084 & 2.71 & Up & \\
\hline hsa-miR-20a & 0.035924 & 0.135700 & 2.69 & Up & \\
\hline hsa-miR-502-3p & 0.000625 & 0.032735 & 2.60 & Up & \\
\hline hsa-miR-7-1* & 0.002495 & 0.054656 & 2.59 & Up & \\
\hline hsa-miR-3677 & 0.014330 & 0.089658 & 2.55 & Up & \\
\hline hsa-miR-1274b & 0.027516 & 0.119268 & 2.49 & Up & \\
\hline hsa-miR-218-2* & 0.004335 & 0.064409 & 2.49 & Up & \\
\hline hsa-miR-18a & 0.006475 & 0.071578 & 2.38 & Up & \\
\hline hsa-miR-330-3p & 0.004056 & 0.064314 & 2.36 & Up & \\
\hline hsa-miR-1274a & 0.004302 & 0.064409 & 2.32 & Up & \\
\hline hsa-miR-29a & 0.005922 & 0.070658 & 2.31 & Up & \\
\hline hsa-miR-138-1* & 0.000786 & 0.039488 & 2.30 & Up & \\
\hline hsa-miR-744 & 0.000059 & 0.015689 & 2.28 & Up & \\
\hline hsa-miR-132 & 0.007974 & 0.075066 & 2.19 & Up & \\
\hline hsa-miR-1181 & 0.000288 & 0.024545 & 2.19 & Up & \\
\hline hsa-miR-29c & 0.026647 & 0.117616 & 2.17 & Up & \\
\hline hsa-miR-3195 & 0.029339 & 0.121421 & 2.16 & Up & \\
\hline
\end{tabular}


Table 2 continued

\begin{tabular}{|c|c|c|c|c|c|}
\hline miRNA & $P$ value & Corrected $P$ value & Fold change & Regulation & AUC \\
\hline hsa-miR-24 & 0.007116 & 0.073136 & 2.13 & Up & \\
\hline hsa-miR-3125 & 0.004598 & 0.064409 & 2.10 & Up & \\
\hline hsa-miR-3127 & 0.004650 & 0.064409 & 2.05 & Up & \\
\hline hsa-miR-192* & 0.000160 & 0.022287 & 2.04 & Up & \\
\hline hsa-miR-1288 & 0.004046 & 0.064314 & 2.03 & Up & \\
\hline hsa-miR-200c & 0.000842 & 0.040212 & 2.01 & Up & \\
\hline hsa-miR-3653 & 0.000338 & 0.024640 & 9.35 & Down & \\
\hline hsa-miR-181d & 0.001044 & 0.040600 & 6.90 & Down & \\
\hline hsa-miR-1268 & 0.001753 & 0.044227 & 4.61 & Down & \\
\hline hsa-miR-222 & 0.011501 & 0.083679 & 4.47 & Down & \\
\hline hsa-miR-623 & 0.007440 & 0.073136 & 4.14 & Down & \\
\hline hsa-miR-574-5p & 0.000088 & 0.017647 & 3.58 & Down & \\
\hline hsa-miR-92a & 0.000166 & 0.022287 & 3.37 & Down & \\
\hline hsa-miR-99b & 0.025374 & 0.114210 & 3.36 & Down & \\
\hline hsa-miR-548f & 0.000032 & 0.015689 & 3.06 & Down & \\
\hline hsa-miR-564 & 0.003564 & 0.063109 & 2.88 & Down & \\
\hline hsa-miR-320a & 0.025932 & 0.115734 & 2.87 & Down & \\
\hline hsa-miR-206 & 0.000138 & 0.022287 & 2.80 & Down & \\
\hline hsa-miR-181a & 0.017001 & 0.093166 & 2.78 & Down & \\
\hline hsa-miR-3663-5p & 0.014980 & 0.089808 & 2.77 & Down & \\
\hline hsa-miR-595 & 0.005164 & 0.068567 & 2.66 & Down & \\
\hline hsa-miR-942 & 0.029072 & 0.121421 & 2.65 & Down & \\
\hline hsa-miR-494 & 0.003770 & 0.063109 & 2.61 & Down & \\
\hline hsa-miR-423-5p & 0.007923 & 0.075066 & 2.59 & Down & \\
\hline hsa-miR-142-5p & 0.025401 & 0.114210 & 2.52 & Down & \\
\hline hsa-miR-1228* & 0.000065 & 0.015689 & 2.52 & Down & \\
\hline hsa-miR-532-3p & 0.016610 & 0.093166 & 2.41 & Down & \\
\hline hsa-miR-670 & 0.001332 & 0.044227 & 2.41 & Down & \\
\hline hsa-miR-3191 & 0.007583 & 0.073136 & 2.38 & Down & \\
\hline hsa-miR-452* & 0.003478 & 0.062543 & 2.35 & Down & \\
\hline hsa-miR-940 & 0.001816 & 0.044648 & 2.28 & Down & \\
\hline hsa-miR-30c & 0.001368 & 0.044227 & 2.27 & Down & \\
\hline hsa-miR-4316 & 0.013200 & 0.087753 & 2.25 & Down & \\
\hline hsa-miR-145 & 0.010675 & 0.082458 & 2.25 & Down & \\
\hline hsa-miR-1306 & 0.005642 & 0.068843 & 2.19 & Down & \\
\hline hsa-miR-186 & 0.007169 & 0.073136 & 2.15 & Down & \\
\hline hsa-miR-3147 & 0.000301 & 0.024545 & 2.14 & Down & \\
\hline hsa-miR-425 & 0.005463 & 0.068567 & 2.08 & Down & \\
\hline hsa-miR-3686 & 0.007328 & 0.073136 & 2.08 & Down & \\
\hline hsa-miR-874 & 0.023395 & 0.112764 & 2.02 & Down & \\
\hline hsa-miR-920 & 0.029734 & 0.121421 & 2.01 & Down & \\
\hline
\end{tabular}

TOF-all all patients with Tetralogy of Fallot, TOF-nOHF TOF patients without heart failure, TOF-HF TOF patients with heart failure, AUC area under the receiver operating characteristic curve

* $P \leq 0.05$

for six miRNAs namely miR-181d-5p, miR-142-5p, miR1233-3p, miR-206, miR-339-5p and miR-625-5p. The significance of the differences in the expression was confirmed for four of the miRNAs, including three downregulated miRNAs namely miR-181d-5p, miR-140-3p and miR-206 and one up-regulated miRNA namely miR625-5p $(P<0.05)$ (Fig. 1). In the second validation step, a total of 34 TOF-noHF patients and 15 control samples were included. The RT-qPCR showed the same direction of expression changes as the microarray analysis for five 


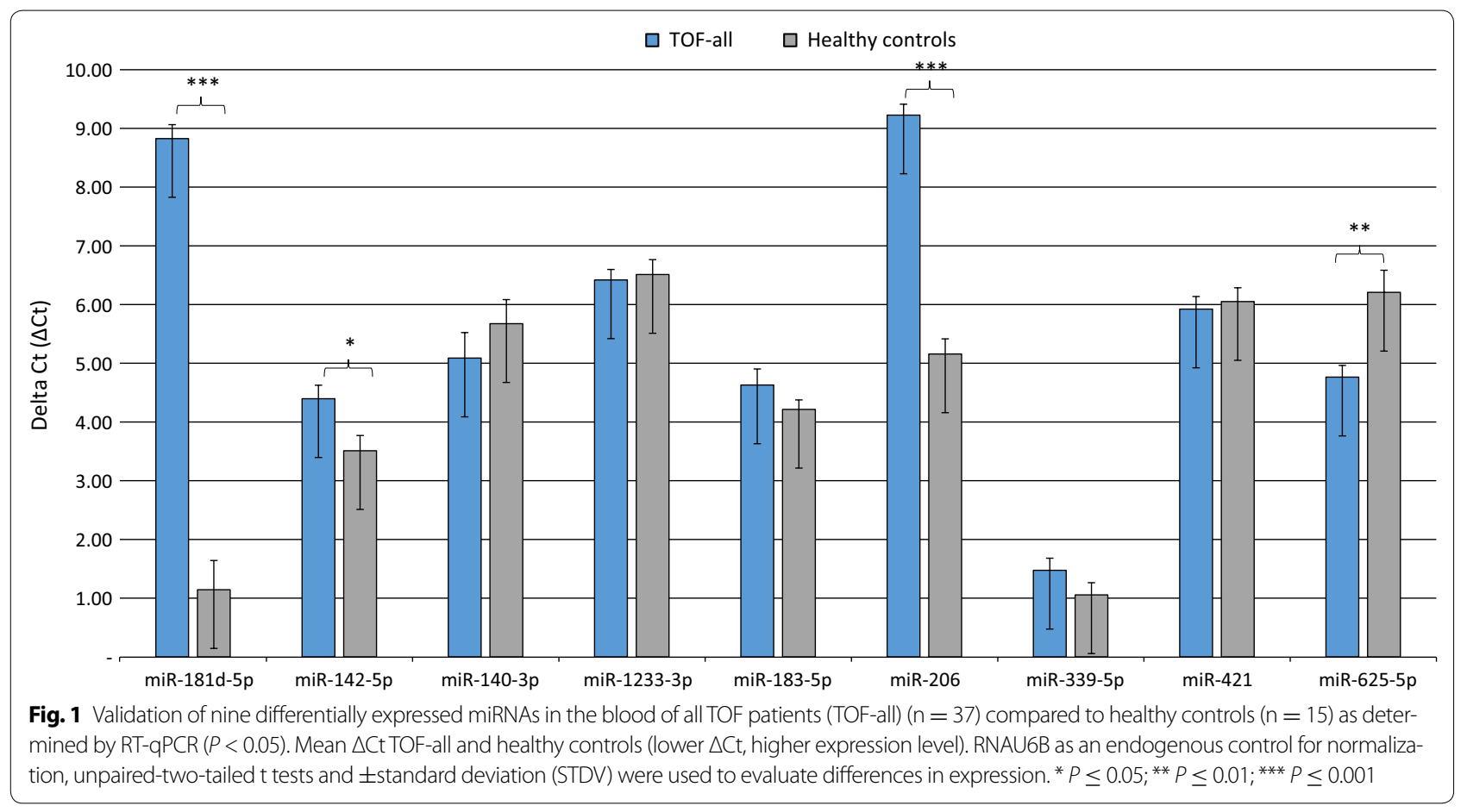

miRNAs namely miR-181d-5p, miR-142-5p, miR-206, miR-339-5p and miR-625-5p. The significance of the differences in the expression was confirmed for four of the miRNAs, including three down-regulated miRNAs namely miR-181d-5p, miR-140-3p and miR-206 and one up-regulated miRNA namely miR-625-5p $)(P<0.05)$
(Fig. 2). In the third validation step, a total of 3 TOF-HF patients and matched controls were included. The RTqPCR showed the same direction of expression changes as the microarray analysis for six miRNAs namely miR181d-5p, miR-1233-3p, miR-183-5p, miR-206, miR-421 and miR-625-5p. The significance of the differences in the

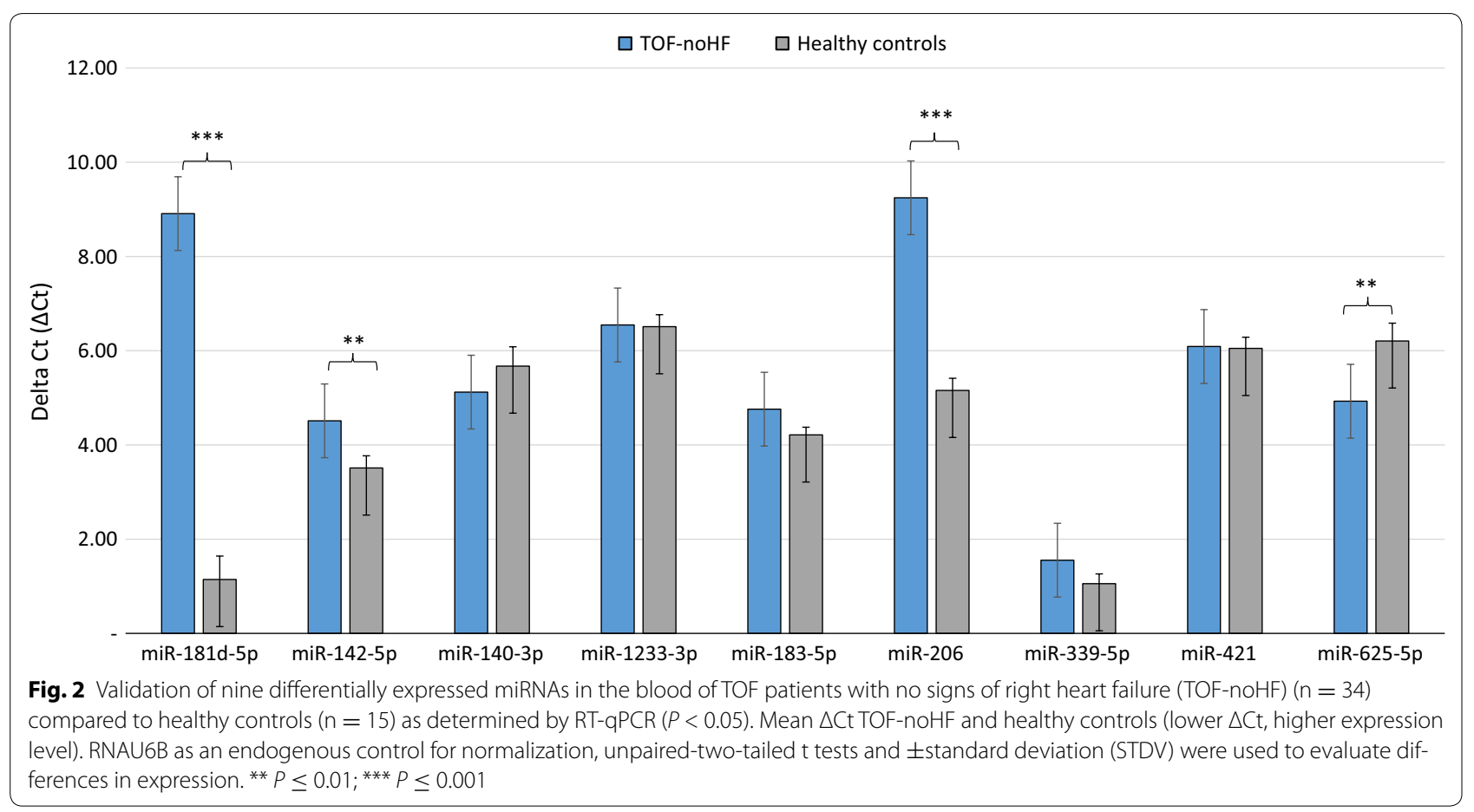


expression was confirmed for six of the miRNAs, including two down-regulated miRNAs namely miR-181d-5p and miR-206 and four up-regulated miRNA namely miR-1233-3p, miR-183-5p, miR-421 and miR-625-5p) $(P<0.05)$ (Fig. 3). Together, the three miRNAs namely miR-181d-5p, miR-206 and miR-625-5p were significantly deregulated in all subgroups of TOF patients.

\section{Relation to clinical variables}

No correlations were found between six of the nine validated miRNAs by RT-qPCR and various clinical parameters such as NYHA class, blood pressure or transcutaneous oxygen saturation. However, weak correlations were observed between miR-421 and miR-1233-5p and right ventricular volumes, ejection fraction as well as hsTNT (Table 3). Moreover, differential expression levels of miR-421, miR-1233-3p and miR-625-5p were found in TOF-noHF and TOF-HF patients with significantly reduced expression levels in TOF patients with symptomatic right heart failure (Fig. 4). No differences in expression levels of these 3 miRNAs were seen for the presence of residual lesions associated with pressure or volume overload of the right ventricle.

\section{Diagnostic accuracy of the validated miRNAs}

To evaluate whether specific miRNAs might be capable of discriminating patients from controls, ROC curve analysis was performed for the validated three miRNAs between TOF-all patients, TOF-noHF patients and healthy controls. Compared with controls, the AUCs
Table 3 Relation of miR-421 and miR-1233-3p with different variables in all TOF patients

\begin{tabular}{|c|c|c|c|c|}
\hline Variables & $\begin{array}{l}\mathrm{miR}-421 \\
r\end{array}$ & $P$ value & $\begin{array}{l}\text { miR-1233-3p } \\
r\end{array}$ & $P$ value \\
\hline Age at follow-up (year) & 0.258 & NS & 0.158 & NS \\
\hline NYHA functional class & -0.175 & NS & -0.120 & NS \\
\hline Systolic blood pressure & -0.097 & NS & 0.020 & NS \\
\hline Diastolic blood pressure & 0.081 & NS & 0.072 & NS \\
\hline $\begin{array}{l}\text { Transcutaneous oxygen } \\
\text { saturation at rest }\end{array}$ & 0.157 & NS & 0.168 & NS \\
\hline Ejection fraction of RV & 0.398 & 0.02 & 0.403 & 0.018 \\
\hline Enddiastolic volume of RV & -0.364 & 0.034 & -0.384 & 0.025 \\
\hline Endsystolic volume of RV & -0.405 & 0.017 & -0.411 & 0.016 \\
\hline Ejection fraction of LV & 0.030 & NS & 0.008 & NS \\
\hline Enddiastolic volume of LV & -0.282 & NS & -0.235 & NS \\
\hline Endsystolic volume of LV & -0.264 & NS & -0.230 & NS \\
\hline VTI above aortic valve & 0.278 & NS & 0.295 & NS \\
\hline NT-proBNP & 0.110 & NS & 0.059 & NS \\
\hline High sensitive Troponin T & -0.488 & 0.003 & -0.493 & 0.002 \\
\hline
\end{tabular}

Spearman rank correlation

NYHA New York Heart Association, RV right ventricle, LV left ventricle, VTI velocity time integral, NS not significant

for miR-181d-5p, miR-206 and miR-625-5p in TOF-all patients were $0.9874,0.9928$ and 0.7694 , respectively and in TOF-noHF patients $0.9902,0.9941$ and 0.7490 , respectively. Together, these results indicate that these three identified miRNAs can discriminate between TOF patients and healthy controls with high accuracy.

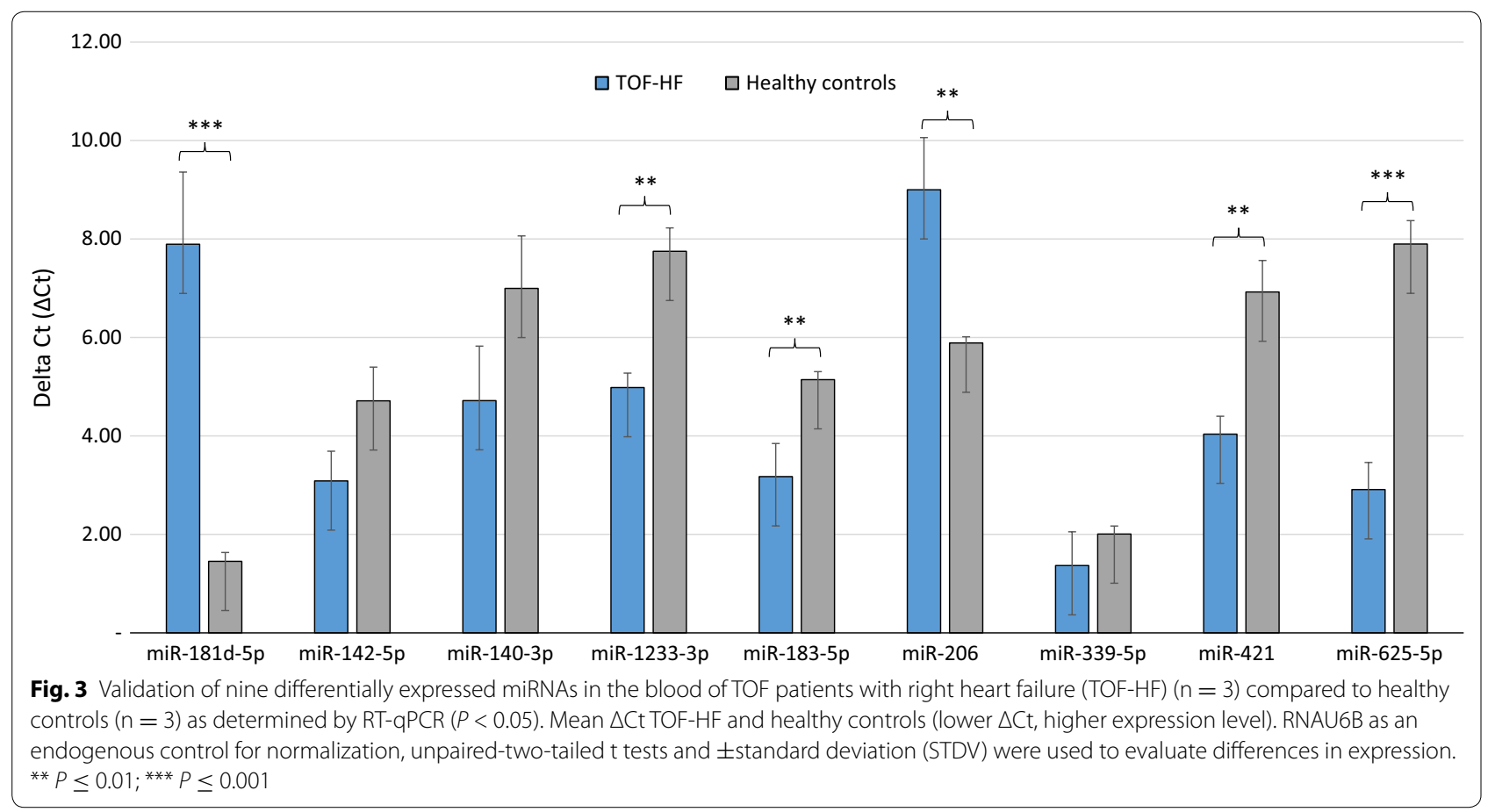




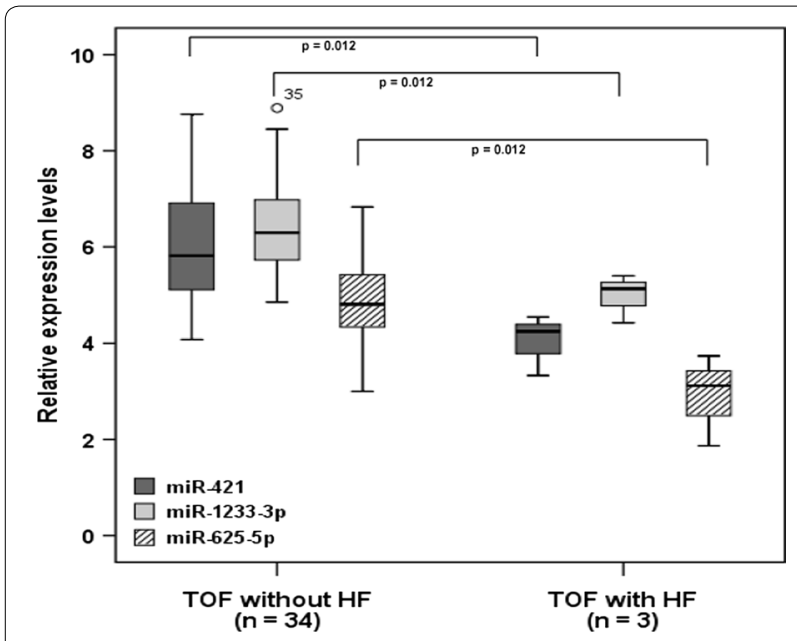

Fig. 4 Boxplots representing relative expression levels of miR-421, miR-1233-3p and miR-625-5p in TOF patients with and without symptomatic right heart failure

\section{Comparative pathway analysis}

To gain insights into the potential impact of the three validated miRNAs (miR-181d-5p, miR-206 and miR625-5p) in regulating target genes, we applied miRTargetLink [23]. Our analysis suggested a tripartite network, containing besides the 3 miRNAs, 11 genes that are targeted by two or more of the selected miRNAs in the 'Strong + Weak' category. Strong interaction was observed between miR-181d-5p and BCL2 and is highlighted in 'Green' in the resulting network (Additional file 3: Figure S2).

\section{Discussion}

MiRNAs are known to be involved in various pathophysiologies and cardiovascular disease including CHD [8, 14, $15,17]$. Recent studies have shown that specific patterns of miRNAs are specifically involved in the development of CHD and TOF $[9,13]$ and that expression levels of altered miRNAs may also be associated with measures of ventricular function [16] or even disease progression and outcome [35]. In this study, we identified an altered miRNA expression profile in TOF patients with and without symptomatic right heart failure when compared with age-matched healthy controls by microarray and further validated by RT-qPCR analyses. The diagnostic accuracy of only three of the validated miRNAs namely miR181d-5p, miR-206 and miR-625-5p by ROC analysis was high with AUCs of $0.987,0.993$ and 0.769 respectively in all TOF patients and $0.990,0.994$ and 0.749 respectively in the subset of TOF patients without symptomatic right heart failure. These results indicate the high diagnostic accuracy of miR-181d-5p and miR-206 and a moderate accuracy of miR-625-5p in differentiating TOF patients from healthy controls. It is of note, however, that miR625-5p showed the highest fold change between healthy controls and the subset of TOF patients with symptomatic right heart failure. The miRNA expression profile found in the blood of surgically repaired TOF patients is different to that found in RVOT tissue of infants with TOF $[9,10]$. In the latter group, altered expression levels of miR-421, miR-1275, miR-27b, miR-1201 and miR-122 have been reported. MiR-940 was found as most downregulated and miR-204 as most up-regulated miRNA [9, $10,13]$. However, miRNA in myocardial tissue of infants undergoing surgical repair reflects a completely different situation than circulating miRNAs in TOF patients late after surgical repair usually associated with the presence of different residual lesions and loading conditions of the right ventricle. Nevertheless, miR-421 was also significantly altered in the blood of patients after long-term repair of TOF suggesting a specific role of miR-421 in the early pathology of TOF as well as during long-term follow-up. For further analysis of the patient group, we additionally selected three miRNAs (miR-1233-3p, miR140-3p and miR-421) with low or moderate expression levels in the three comparisons and miR-421 that had been identified in myocardial tissue of TOF patients [9]. We found that expression levels of circulating miR-421, miR-1233-3p and miR-625-5p were significantly lower in TOF patients with as compared to those without symptomatic right heart failure indicating a potential role of these miRNAs in identifying disease progression in TOF patients. However, no differences in expression levels of these miRNAs were seen for the presence of residual lesions associated with right ventricular pressure overload such as significant restenosis of the RVOT or right ventricular volume overload due to significant pulmonary regurgitation. In our patient group, symptomatic right heart failure was also associated with left ventricular dysfunction as indicated by the reduced left ventricular ejection fraction, velocity time integral above the aortic valve and markedly elevated NT-proBNP levels. It is known from previous studies that LV dysfunction may also be present late after TOF repair due to various reasons such as RV enlargement/dysfunction associated with interventricular mechanical or electrical dyssynchrony [3, 36, 37] or longstanding cyanosis prior to corrective surgery [2]. This is consistent with our findings of increased right ventricular volumes and older age at corrective surgery in the TOF-HF group. Recently, it has been shown that levels of circulating miRNAs are declining with progression or increasing acuity of heart failure in patients with left heart failure [35]. Although a different expression profile was found in these patients, the results of our study with lower miRNA expression levels found in TOF-HF patients are in agreement with that study and 
thus may also be indicative of disease progression in TOF patients. However, these results should be confirmed in a larger cohort of patients, especially in the TOF-HF subset. Bioinformatics analysis helped to gain insights into the potential impact of the three validated miRNAs (miR-181d-5p, miR-206 and miR-625-5p) on target genes (Additional file 3: Figure S2). Among the predicted target genes was B-cell lymphoma 2 (Bcl-2), which plays a critical role in the inhibition of apoptosis [38], Bcl-2 expressed in myocytes of the human heart with infarction and participates in the protection or acceleration of cellular damage after infarction [39]. Similarly, Insulinlike growth factor 1 receptor (IGF1R), protects the heart in settings of heart diseases [40] and plays a role in proliferation, cardiac differentiation, and decreases apoptosis in human heart [41]. Bromodomain Adjacent to Zinc Finger Domain 2A (BAZ2A), plays an important role in the transcription deregulation in hypertrophy and heart failure [42].

\section{Conclusion}

Altered expression levels of circulating miRNAs can be found in TOF patients late after surgical repair and are different to those seen in the right ventricular myocardium of infants with TOF. Expression levels of miR-421, miR-1233-3p and miR-625-5p are lower in TOF patients with symptomatic right heart failure and thus may indicate disease progression in these patients.

\section{Additional files}

Additional file 1: Table S1. Expression of all significantly miRNAs in the blood samples of TOF-all, TOF-noHF, and TOF-HF patients compared with those of controls (age-matched) as determined by microarray (Unpaired two-tailed $t$ test, $P<0.05)$.

Additional file 2: Figure S1. Unsupervised hierarchical clustering (Euclidian distance, complete linkage) of the patients compared to matched controls based on expression of the 50 with the highest variance. $1 \mathrm{~A}$ ) the first cluster contains mostly controls and the second most of the TOF-HF patients. 1B) and 1C) A more detailed distinction between the TOF-noHF and TOF-all subset, each matched to controls based on the clustering dendrogram were, however, not conclusive.

Additional file 3: Figure S2. Target network: for the three validated miRNAs in brown, target genes are presented in blue.

\footnotetext{
Abbreviations

BAZ2A: adjacent to zinc finger domain 2A; BCl-2: B-cell lymphoma 2; CHD: congenital heart defect; DMSO: dimethyl sulfoxide; GAPDH: glyceraldehyde 3-phosphate dehydrogenase; IGF1R: insulin-like growth factor 1 receptor; LV: left ventricle; miRNA: microRNA; NS: not significant; NT-proBNP: N-terminal pro B-type natriuretic peptide; NYHA: New York Heart Association; RT-qPCR: quantitative reverse transcription-polymerase chain reaction; RV: right ventricle; RVOT: right ventricular outflow tract; TGS: total gene signals; TOF: Tetralogy of Fallot; TOF-all: Tetralogy of Fallot-all patients; TOF-HF: Tetralogy of Fallot patients with symptomatic right heart failure; TOF-noHF: Tetralogy of Fallot patients without symptomatic right heart failure; VSD: ventricular septal defect; $\vee T 1$ : velocity time integral.
}

\section{Authors' contributions}

MA, performed experimental work, particularly the miRNA isolation, array experiment, RT-qPCR validation and helped in manuscript writing; EM, designed the study, coordinated the molecular biology experiment and edited the manuscript; AK, performed bioinformatics analysis; HAK, designed the study and diagnosed patients; TRH, designed the study, recruited and examined controls, diagnosed patients, collected blood samples and helped in writing. All authors read and approved the final manuscript.

\section{Author details}

1 Department of Human Genetics, Saarland University, 66421 Homburg/ Saar, Germany. ${ }^{2}$ Chair for Clinical Bioinformatics, Saarland University, 66041 Saarbruecken, Germany. ${ }^{3}$ Department of Pediatric Cardiology, Saarland University Medical Center, 66421 Homburg/Saar, Germany. ${ }^{4}$ Department of Human Genetics, Saarland University Medical Center, Kirrberger Straße 100, 66421 Homburg/Saar, Germany.

\section{Acknowledgements \\ Not applicable.}

\section{Competing interests}

The authors declare that they have no competing interests.

\section{Availability of data and materials}

The data sets during and/or analyzed during the current study available from the corresponding author on reasonable request.

\section{Consent for publication}

Subjects were recruited and evaluated in accordance with relevant guidelines and regulations following the approval of the local ethics committee (Ethical vote No. 73/09). All participants or their legal guardians gave written informed consent before enrolment.

\section{Ethics approval and consent to participate}

Institutional Review Board approval/Ethikvotum Ärztekammer des Saarlandes: Ethical vote No. 73/09.

\section{Funding}

This study was funded by the Fördergemeinschaft Kinderherzen in Bonn, the Competence Network for Congenital Heart Defects, which received funding from the Federal Ministry of Education and Research, Grant Number 01 Gl0601 (2014), and the German Centre for Cardiovascular Research (DZHK), Grant Number $81 \times 2800112$ (2015)

\section{Publisher's Note}

Springer Nature remains neutral with regard to jurisdictional claims in published maps and institutional affiliations.

Received: 24 March 2017 Accepted: 24 June 2017

Published online: 10 July 2017

\section{References}

1. Hoffman JI, Kaplan S. The incidence of congenital heart disease. J Am Coll Cardiol. 2002;39:1890-900.

2. Broberg CS, Aboulhosn J, Mongeon FP, Kay J, Valente AM, Khairy P, Earing MG, Opotowsky AR, Lui G, Gersony DR, et al. Prevalence of left ventricular systolic dysfunction in adults with repaired Tetralogy of Fallot. Am J Cardiol. 2011;107:1215-20.

3. Cheung EW, Liang XC, Lam WW, Cheung YF. Impact of right ventricular dilation on left ventricular myocardial deformation in patients after surgical repair of Tetralogy of Fallot. Am J Cardiol. 2009;104:1264-70.

4. Latus H, Hachmann P, Gummel K, Khali M, Yerebakan C, Bauer J, Schranz D, Apitz C. Impact of residual right ventricular outflow tract obstruction on biventricular strain and synchrony in patients after repair of Tetralogy of Fallot: a cardiac magnetic resonance feature tracking study. Eur J Cardiothorac Surg. 2015;48:83-90. 
5. Shin YR, Jung JW, Kim NK, Choi JY, Kim YJ, Shin HJ, Park YH, Park HK. Factors associated with progression of right ventricular enlargement and dysfunction after repair of Tetralogy of Fallot based on serial cardiac magnetic resonance imaging. Eur J Cardiothorac Surg. 2016;50:464-9.

6. Tzemos N, Harris L, Carasso S, Subira LD, Greutmann M, Provost Y, Redington AN, Rakowski H, Siu SC, Silversides CK. Adverse left ventricular mechanics in adults with repaired Tetralogy of Fallot. Am J Cardiol. 2009;103:420-5.

7. Pasquinelli AE. MicroRNAs and their targets: recognition, regulation and an emerging reciprocal relationship. Nat Rev Genet. 2012;13:271-82.

8. Smith T, Rajakaruna C, Caputo M, Emanueli C. MicroRNAs in congenital heart disease. Ann Transl Med. 2015;3:333.

9. Bittel DC, Kibiryeva N, Marshall JA, O'Brien JE. MicroRNA-421 dysregulation is associated with Tetralogy of Fallot. Cells. 2014;3:713-23.

10. O'Brien JE Jr, Kibiryeva N, Zhou XG, Marshall JA, Lofland GK, Artman M, Chen J, Bittel DC. Noncoding RNA expression in myocardium from infants with Tetralogy of Fallot. Circ Cardiovasc Genet. 2012;5:279-86.

11. Wang XM, Zhang K, Li Y, Shi K, Liu YL, Yang YF, Fang Y, Mao M. Screening miRNA and their target genes related to Tetralogy of Fallot with microarray. Cardiol Young. 2014;24:442-6.

12. Zhang J, Chang JJ, Xu F, Ma XJ, Wu Y, Li WC, Wang HJ, Huang GY, Ma D. MicroRNA deregulation in right ventricular outflow tract myocardium in nonsyndromic Tetralogy of Fallot. Can J Cardiol. 2013;29:1695-703.

13. Liang D, Xu X, Deng F, Feng J, Zhang H, Liu Y, Zhang Y, Pan L, Liu Y, Zhang D, et al. miRNA-940 reduction contributes to human Tetralogy of Fallot development. J Cell Mol Med. 2014;18:1830-9.

14. Dimmeler S, Zeiher AM. Circulating microRNAs: novel biomarkers for cardiovascular diseases? Eur Heart J. 2010;31:2705-7.

15. Gupta SK, Bang C, Thum T. Circulating microRNAs as biomarkers and potential paracrine mediators of cardiovascular disease. Circ Cardiovasc Genet. 2010;3:484-8.

16. Lai CT, Ng EK, Chow PC, Kwong A, Cheung YF. Circulating microRNA expression profile and systemic right ventricular function in adults after atrial switch operation for complete transposition of the great arteries. BMC Cardiovasc Disord. 2013;13:73.

17. Li D, Ji L, Liu L, Liu Y, Hou H, Yu K, Sun Q, Zhao Z. Characterization of circulating microRNA expression in patients with a ventricular septal defect. PLOS ONE. 2014:9:e106318.

18. Zhu S, Cao L, Zhu J, Kong L, Jin J, Qian L, Zhu C, Hu X, Li M, Guo X, et al. Identification of maternal serum microRNAs as novel non-invasive biomarkers for prenatal detection of fetal congenital heart defects. Clin Chim Acta. 2013:424:66-72.

19. Tijsen AJ, Creemers EE, Moerland PD, de Windt $L$, van der Wal AC, Kok WE, Pinto YM. MiR423-5p as a circulating biomarker for heart failure. Circ Res. 2010;106:1035-9.

20. Tutarel O, Dangwal S, Bretthauer J, Westhoff-Bleck M, Roentgen P, Anker SD, Bauersachs J, Thum T. Circulating miR-423_5p fails as a biomarker for systemic ventricular function in adults after atrial repair for transposition of the great arteries. Int J Cardiol. 2013;167:63-6.

21. Abu-Halima M, Ludwig N, Hart M, Leidinger P, Backes C, Keller A, Hammadeh M, Meese E. Altered micro-ribonucleic acid expression profiles of extracellular microvesicles in the seminal plasma of patients with oligoasthenozoospermia. Fertil Steril. 2016;106:1061.

22. Livak KJ, Schmittgen TD. Analysis of relative gene expression data using real-time quantitative $P C R$ and the $2(-\triangle \triangle C(T))$ method. Methods 2001;25:402-8.

23. Hamberg M, Backes C, FehImann T, Hart M, Meder B, Meese E, Keller A. MiRTargetLink—-miRNAs, genes and interaction networks. Int J Mol Sci. 2016:17:564.

24. Ellis KL, Cameron VA, Troughton RW, Frampton CM, Ellmers LJ, Richards AM. Circulating microRNAs as candidate markers to distinguish heart failure in breathless patients. Eur J Heart Fail. 2013;15:1138-47.
25. Kumar S, Kim CW, Simmons RD, Jo H. Role of flow-sensitive microRNAs in endothelial dysfunction and atherosclerosis: mechanosensitive atheromiRs. Arterioscler Thromb Vasc Biol. 2014;34:2206-16.

26. Kumarswamy R, Thum T. Non-coding RNAs in cardiac remodeling and heart failure. Circ Res. 2013;113:676-89.

27. Kuosmanen SM, Hartikainen J, Hippelainen M, Kokki H, Levonen AL, Tavi P. MicroRNA profiling of pericardial fluid samples from patients with heart failure. PLOS ONE. 2015;10:e0119646.

28. Li H, Fan J, Yin Z, Wang F, Chen C, Wang DW. Identification of cardiacrelated circulating microRNA profile in human chronic heart failure. Oncotarget. 2016;7:33-45.

29. Liu Y, Chen S, Zhang J, Shan S, Chen L, Wang R, Kan J, Xu T. Analysis of serum microRNAs as potential biomarker in coronary bifurcation lesion. Dis Markers. 2015;2015:351015.

30. Marques FZ, Vizi D, Khammy O, Mariani JA, Kaye DM. The transcardiac gradient of cardio-microRNAs in the failing heart. Eur J Heart Fail. 2016;18:1000-8.

31. Saddic LA, Chang TW, Sigurdsson MI, Heydarpour M, Raby BA, Shernan SK, Aranki SF, Body SC, Muehlschlegel JD. Integrated microRNA and mRNA responses to acute human left ventricular ischemia. Physiol Genomics. 2015;47:455-62.

32. Schulte C, Westermann D, Blankenberg S, Zeller T. Diagnostic and prognostic value of circulating microRNAs in heart failure with preserved and reduced ejection fraction. World J Cardiol. 2015;7:843-60.

33. Schulte C, Zeller T. microRNA-based diagnostics and therapy in cardiovascular disease-summing up the facts. Cardiovasc Diagn Ther. 2015;5:17-36.

34. Wong LL, Armugam A, Sepramaniam S, Karolina DS, Lim KY, Lim JY, Chong JP, Ng JY, Chen YT, Chan MM, et al. Circulating microRNAs in heart failure with reduced and preserved left ventricular ejection fraction. Eur J Heart Fail. 2015:17:393-404.

35. Ovchinnikova ES, Schmitter D, Vegter EL, Ter Maaten JM, Valente MA, Liu LC, van der Harst P, Pinto YM, de Boer RA, Meyer S, et al. Signature of circulating microRNAs in patients with acute heart failure. Eur I Heart Fail. 2016:18:414-23.

36. Kempny A, Diller GP, Orwat S, Kaleschke G, Kerckhoff G, Bunck A, Maintz $D$, Baumgartner $\mathrm{H}$. Right ventricular-left ventricular interaction in adults with Tetralogy of Fallot: a combined cardiac magnetic resonance and echocardiographic speckle tracking study. Int J Cardiol. 2012;154:259-64.

37. Raedle-Hurst TM, Mueller M, Rentzsch A, Schaefers HJ, Herrmann E, Abdul-Khaliq H. Assessment of left ventricular dyssynchrony and function using real-time 3-dimensional echocardiography in patients with congenital right heart disease. Am Heart J. 2009;157:791-8.

38. Allsopp TE, Wyatt S, Paterson HF, Davies AM. The proto-oncogene bcl-2 can selectively rescue neurotrophic factor-dependent neurons from apoptosis. Cell. 1993;73:295-307.

39. Misao J, Hayakawa Y, Ohno M, Kato S, Fujiwara T, Fujiwara H. Expression of $\mathrm{bcl}-2$ protein, an inhibitor of apoptosis, and Bax, an accelerator of apoptosis, in ventricular myocytes of human hearts with myocardial infarction. Circulation. 1996;94:1506-12.

40. McMullen JR, Shioi T, Huang WY, Zhang L, Tarnavski O, Bisping E, Schinke M, Kong S, Sherwood MC, Brown J, et al. The insulin-like growth factor 1 receptor induces physiological heart growth via the phosphoinositide 3-kinase(p110a) pathway. J Biol Chem. 2004;279:4782-93.

41. D'Amario D, Cabral-Da-Silva MC, Zheng H, Fiorini C, Goichberg P, Steadman E, Ferreira-Martins J, Sanada F, Piccoli M, Cappetta D, et al. Insulin-like growth factor-1 receptor identifies a pool of human cardiac stem cells with superior therapeutic potential for myocardial regeneration. Circ Res. 2011:108:1467-81.

42. Sucharov CC, Sucharov J, Karimpour-Fard A, Nunley K, Stauffer BL, Miyamoto SD. Micro-RNA expression in hypoplastic left heart syndrome. J Card Fail. 2015:21:83-8. 